\title{
A New Point of Consideration for the Definition of Minor Ischemic Stroke: Review
}

\author{
Angela P. Fernandes*, Sandip Kumar Jaiswal, Fu-Ling Yan \\ Department of Neurology, Zhongda Hospital Affiliated to Southeast University, Nanjing, China \\ Email: *ap.fernandes@live.com.pt
}

How to cite this paper: Fernandes, A.P., Jaiswal, S.K. and Yan, F.-L. (2021) A New Point of Consideration for the Definition of Minor Ischemic Stroke: Review. Neuroscience \& Medicine, 12, 44-54. https://doi.org/10.4236/nm.2021.121004

Received: February 2, 2021

Accepted: March 23, 2021

Published: March 26, 2021

Copyright (อ 2021 by author(s) and Scientific Research Publishing Inc. This work is licensed under the Creative Commons Attribution International License (CC BY 4.0).

http://creativecommons.org/licenses/by/4.0/ (c) (i) Open Access

\begin{abstract}
Stroke is the second highest cause of death globally and a leading cause of not only physical disability but also cognitive. Approximately two-thirds of Ischemic Stroke patients, who represent the most common type of stroke are found to have mild deficits. Minor stroke, sometimes also referred to as "mild stroke", is often defined in research studies as a National Institute of Health Stroke Scale (NIHSS) score of 5 or less, although, the cut point for NIHSS score or standardized criterion to define minor stroke has not been established. In the past, many studies have been focusing on the definition of the minor stroke to better stabilize the acute treatment guidelines, to predict the functional outcome, and also for early risk factors stratification. Different studies use different criteria or different cut-points of NIHSS scores to define minor stroke. Aside from indecision of acute management of minor stroke, many other questions have been raised regarding minor strokes, for example, the interaction of large vessel occlusion with minor stroke, the prevalence rate of depression, anxiety, cognitive dysfunction, chronic head after minor stroke, and so on. Finding a universal definition for minor stroke is the key to establish the guideline for management for this group of patients. However, the guidance of treatment of minor stroke is not the focus of this review. The review will focus on the deep comprehension of minor stroke characteristics, summarizing the new findings related to minor stroke, and highlight the essential points to consider for characterizing mild stroke symptoms for a new direction guide for future studies.
\end{abstract}

\section{Keywords}

Mild Stroke, Risk Factors, NIHSS Score, Large Vessel Occlusion, Depression, Chronic Headache, Cognitive Dysfunction 


\section{Introduction/Background}

\section{Definition}

The term "minor", as an adjective, is referred to as inferior to importance, size, degree, or seriousness, considering this definition of the term "minor", we are assuming that all minor stroke occurrences present whit a small degree of disability, less seriousness or importance. Perhaps, it is necessary to establish what should be considered minor, and what should not be.

Determining one unanimous definition for minor stroke has been a challenge for researchers. Up until now, the universal standard definition for minor stroke has not been established yet, and the controversy about the acute management remains [1] [2]. Previously, many stroke tools (e.g., NIHSS-National Institute of Health Stroke Scale, SSS-Scandinavian Stroke Scale, mRS-modified Rankin Scale, BI-Barthel Index, SIS-Stroke Impact Scale Table 1) have been a subject of study for better comprehension of minor stroke, with an objective to obtain a consensus on the definition of the minor stroke. The most commonly accepted definition of minor stroke was based on the cutoff NIHSS score or consideration of some stroke symptoms. However, a definition of minor stroke will depend on the point of interest of the researchers. Some studies choose to use the absence or

Table 1. Scales used in clinical interpretation of stroke.

\section{Stroke Tools and Application}

\section{NIHSS (National Institute of Health Stroke Scale)}

Is a systematic assessment tool that provides a quantitative measure of stroke-related neurologic deficit. A different cutoff NIHSS score are used to classify stroke level of severity: Very Severe: $>25$; Severe: 15 - 24; Mild to Moderately Severe: 5 - 14; Mild: 1 - 5.

\section{SSS (Scandinavian Stroke Scale)}

Is an alternative stroke scale that is designed to give a score based on the level of consciousness eye movement and severity of paralysis. Stroke severity has been graded using this scale as: Mild: 43 58; Moderate: 26 - 42, Severe: 0 - 25.

\section{mRS (Modified Rankin Scale)}

Is a stroke tool used as reference for prediction of outcomes; $\mathrm{mRS}>2$ is considered more related with dependency and death after stroke.

\section{BI (Barthel Index)}

The Barthel index is used to assess disability and to monitor changes in disability over time, second most used stroke scale for prediction and outcomes. The normal score is 100, and lower scores indicate greater dependency.

\section{SIS (Stroke impact Scales)}

Is a stroke scale based on self-report, health status measure that evaluates disability and health-related quality of life after stroke. It was designed to assess multidimensional stroke outcomes, including strength, hand function Activities of Daily Living, communication, emotion, memory and thinking, and participation.

Notes: The NIHSS and SIS scales are used in earlier stage of stroke for decision make of acute treatment and to assess recovery/deterioration; The mRS, BI and SIS are used to determine the level of disability and outcomes; (The NIHSS score tools it's been an important tool to define minor stroke not only in the research studies, but also in clinical practice, by selecting a different cutoff in the NIHSS score for classification of minor and non-minor stroke). 
presence of some stroke symptoms to define minor stroke and, some define minor stroke based on the stroke syndromes [3] [4].

Since 1996, with the FDA approval of intravenous tissue plasminogen activator (tPA) for the treatment of acute ischemic stroke, most of the patients included in the studies for determination of the efficacy of this treatment, presented with a significant deficit-an NIHSS $\geq 9$ score [5]. Up to date, the information about the efficacy of this treatment in the group of patients with mild stroke symptoms is still controversial. Afterward, the importance to determine the definition of minor stroke emerges since mild stroke symptoms are considered to be very common criteria for thrombolysis exclusion [6] [7]. One study found that, patients who were considered too mild or were documented to have significant improvement, either remained dependent at hospital discharge or died during hospital admission [2].

We performed an electronic literature search for different definitions for minor stroke, using the terms "minor stroke" and "mild stroke symptoms" definition, summarized in Table 2, since definitions based on stroke symptoms are more subjective to individual opinion, the results showed that, studies that defined mild stroke based on stroke symptoms, even when only mild symptoms were attributed for minor stroke definition, patients still present disability or some level of complication.

Frequently, in literature the NIHSS score and mRS are used to determine the stroke severity and patient selection during the study, and a different cutoff NIHSS scores were used by different research groups. During our research, we found that the cutoff NIHSS score less than or equal to 5 is the most frequently used in the research studies. However, a cutoff NIHSS less than or equal to 3 points was found to be reasonable to define minor stroke in different studies [3] [8], because patients from this group of study showed better outcomes after 90 days. Other studies define minor stroke as $\mathrm{mRS}$ score $\leq 2$ point, considering the fact that $\mathrm{mRS}$ score gives information about death risk and disability and could better point out the needs of the patients after hospital discharge [1]. Several definitions have been proposed by different studies, to be considered as a possible definition for minor stroke however, no consensus definitions were made.

\section{The National Institutes of Health Stroke Scale (NIHSS) Score and Minor Stroke Symptoms}

The National Institutes of Health Stroke Scale (NIHSS) was developed to help physicians assess the neurological impairment to determine the stroke severity and also to select the candidates for thrombolytic therapy. The current NIH stroke scale contains 11 items with a total score ranging from 0 to 42 points. Up until now the National Institute of Health Stroke Scale (NIHSS) has been considered the most standard and accurate scale used to define minor stroke [1], however, some items of the NIHSS have lower interrater reliability. Hence, many 
Table 2. Definitions for minor stroke-based on the stroke symptoms and outcomes.

Definitions
(Kernan, 1991): Minor Stroke was defined as the
presence of full strength in all but one limb, in
which there could be only a mild weakness, and the
absence of more than mild dysphasia, or patients
with monocular blindness who did not recover
beyond 24 hours [8].

(Carlsson et al., 2004): Mild Stroke was defined as no or slight motor impairment or a high level of independence in personal ADL [9].

\section{Study design/findings}

Retrospective cohort study: The study result showed that, clinical features such as the age of more than 65 years, diabetes, and hypertension, can be combined to stratify effectively the risk for a subsequent stroke or death in patients with minor stroke or TIA.

A qualitative design: This study result showed Astheno-Emotional Syndrome with mental fatiguability as one of the most common symptoms affecting many dimensions of everyday life, which in turn affected the performance of activities and independence.

(Johansson et al., 2007): Mild Stroke was defined as the absence of significant limitations of functions [10].

Quantitative method: Results showed that women with a mild stroke experience ADL problem have the potential to be independent if they received adequate rehabilitation.

(Muren et al., 2008) Mild stroke was defined as the ability to walk more than 10 meters independently, live at home, be independent in activities of daily living [11].

Quantitative method: According to this study, HRQoL was reduced with the lowest SIS scores in the domains of strength, hand function, and participation domains and the highest scores in the domains of mobility, communication, activities of daily living/instrumental activities of daily living, and memory.

(Jones Michael R. et al., 2000): Minor Stroke Retrospective cohort: The study found evidence of a small but significant racial definition was determined by using the absence of motor deficits or presence of mild hemiparesis with or without dysarthria and without alteration in sensation [12].

(National Institute of Neurological Disorders Stroke rt-PA Stroke Study Group, 2005): Minor Stroke was attributed to all patients in the study without measurable deficit on the NIHSS score, pure sensory stroke, isolated ataxia, isolated dysarthria, and isolated facial weakness neglect, horizontal eye movements, or visual fields [13].

(Liu-Ambrose et al., 2007): Minor Stroke was defined as functionally minimal impairments of finer movement but with controlled and normal movement through the full range [14]. difference in initial stroke severity, with black individuals having more severe strokes.

Clinical trial: The result of this clinical trial suggests that the risk-benefit ratio for using tissue plasminogen activator in minor-stroke patients favors treatment in eligible patients.

Abbreviations: The term Mild Stroke = to Minor Stroke; ADL (Activities of Daily Living); HRQoL (Health-Related Quality of life).

controversies are emerging in different studies regarding the accuracy and specificity of the degree of NIHSS score to define minor stroke, highlighting that some items on the NIHS scale are highly weighted, and not all stroke symptoms are captured by the scale. One study showed that a proportion of subjects with TIA or minor stroke who had an NIHSS score of 0 on the evaluation became disabled at 3 months, with $m R S \geq 2$ [15].

Patients with mild stroke symptoms only, should not be underestimated based 
on the low NIHSS score. The following points should be considered when to use NIHSS to define minor stroke. First, the NIHSS is very limited to the aphasic patients, it can be difficult to analyze the degree of deficits. Second, some stroke symptoms are not captured by the NIHSS, for example, ambulation, fine finger movement, distal hand weakness, gait speed, cognitive abnormalities, which can be associated with poor outcomes or stroke deterioration [16] [17]. Third, the difference between left and right hemisphere stroke. Patients with right hemisphere stroke tend to have a lower NIHSS score compared to left hemisphere stroke patients, even though the right hemisphere tends to present a larger size of brain lesion on the imaging [18] [19]. Fourth, symptoms of posterior circulation stroke can be misdiagnosed by NIHSS [20] [21], given that symptoms of posterior circulation such as truncal ataxia, cranial nerve palsies, nausea, headache, dizziness are not captured by NIHSS [15]. Finally, use of NIHSS scores alone to determine stroke severity is very limited, perhaps, an early evaluation of a more specific imaging exam such as MRI-DWI to detect the clear size of brain lesion, vascular imaging of the head and neck to evaluate large artery occlusion can be incorporated to the NIHSS score during stroke evaluation. Other neurological tools such as GCS (Glasgow Coma Scale) can be very useful in patients with posterior circulation stroke to determine the of level consciousness, and tests of swallowing function can also be added to the routine of evaluation of stroke severity since they can be helpful to detect brainstem lesion.

Despite the shortcomings aforementioned, the NIHSS is very reliable to predict poor outcomes in patients with a very high NIHSS score, according to the result of the Trial of Org 10,172 in acute stroke [22]. Another study showed that there is a strong correlation between the NIHSS score and median DWI lesion volume [18] [23]. Different types of neurological deficits may represent a different significance to the patient's ability function. Considering only the NIHSS score to determine stroke severity, can cause neglection of the patient with very significant disabled deficits.

\section{The Interrelationship between Some Specific Stroke Deficits and Minor Stroke}

Considering a stroke symptom as non-disabling is very subjective to the physicians' opinion and, will also, depend on the type of symptoms and which group of patients the symptom is attributed to. For example, some stroke symptoms can be more disabling in old patients compared to young individuals. Concordance has been found between the studies expressing that some stroke deficits such as visual fields, gait ataxia, cranial nerve palsies, neglect, distal hand weakness, dysarthria can predict poor functional outcomes [17] [24] [25]. Another study showed that patients who tested abnormal for the scores on the itemized items for LOC, motor, or ataxia were less likely to be discharged home [16]. However, some specific stroke symptoms were found to be correlated to the poor outcomes in minor ischemic stroke. One study found that $23.8 \%$ of patients 
who presented isolated ataxia had unfavorable outcomes [17]. Another study showed that leg weakness and extinction or inattention symptoms may be also associated with unfavorable outcomes in patients with minor stroke [25]. However, several other symptoms are associated with poor outcomes in minor stroke patients, either independently or associated with others factor such as age, diabetes mellitus, female sex and presence of large vessel occlusion [7] [25] [26].

\section{Minor Stroke and Large Vessel Occlusion}

Up to date, there are no specific guidelines for the management of patients with large vessel occlusion (LVO) who present with mild symptoms. One study showed that about one-third of the ischemic stroke patients harbor large vessel occlusion [27], and the LVO were considered to be very consistent, independent predictor of neurological deterioration and disability in patients with minor stroke [28] [29].

Recently, several clinical trials showed that patients with a large vessel occlusion, often present low NIHSS score, however, they may end up having a poor outcome if left untreated. Different studies found different efficacy of acute management of mild ischemic stroke (NIHSS score $\leq 5$ ) in presence of large vessel occlusion, even though, neither IV thrombolysis nor endovascular therapy (ET) was recommended in the stroke guideline for minor ischemic stroke [30].

Many factors have been hypothesized as contributing to the low NIHSS score on large vessel occlusion stroke. The presence of a good collateral vessel is crucial to determine the stroke severity. Patients with very developed collateral may have mild clinical symptoms during a stroke episode, even if they present a large size lesion in the imaging [31] [32]. LVO in Stroke with low NIHSS score left untreated have a higher chance to develop secondary deterioration [27]. However, studies raised controversies regarding the benefit and efficacy of aggressive management this group of patients with lower NIHSS score. Some studies showed that acute perfusion with primary IVT and/or EVT may be safe and may improve outcomes [29] [31] [33], While, another study found no benefit and with a high risk of serious bleeding [34] [35].

\section{Depression, Anxiety, Cognitive Dysfunction and Headache after Minor Stroke}

Depression after stroke is very common, especially in patients with moderate to severe stroke. Approximately one-third of stroke survivors will suffer from depression at least once [36]. Factors such as physical disability, stroke severity, depression before stroke, cognitive impairment, anxiety, older age, and female sex were found to be consistent prediction for depression after moderate to severe stroke [37] [38]. However, very little is known about the prevalence of depression and cognitive dysfunction in minor stroke patients. One study conducted in patients with minor stroke (cutoff NIHSS score $\leq 5$ ) showed a prevalence rate of $40 \%$ for depression, and components such as low level of education 
and diabetes have a significant correlation with depression after minor ischemic stroke [39]. On the other hand, another study found a prevalence of $29 \%$ depression rate, with a high incidence in the first year after the stroke event [40]. Nevertheless, in this study, the predictors such as female gender, mild global cognitive impairment, smoking, and stroke recurrence, were found to have a strong correlation with depression in minor stroke [40], which is very consistent with some predictors found in more severe stroke.

Minor stroke patients may also have a cognitive function impairment, fatigue, anxiety, chronic headache, and apathy. All these post-stroke complications may have a big impact on the Quality of Life (QOL) [41], social activities, and ability to return to work. Therefore, the early diagnosis and early treatment are crucial to this group of patients, in order to promote a better quality of life. One longitudinal study of cognitive and emotional impairment after a minor stroke showed that there is a significant improvement of cognitive function from 3 to 12 months but prevalence of cognitive impairments in $35 \%$ of the subjects after 12 months [42].

Fatigue may be present even in the absence of mental or functional impairment. One study shows that about $30 \%$ of patients at 6 months, and $34 \%$ at 1 year after a minor stroke may develop fatigue symptoms [43] [44]. Prevalence of anxiety was found to be very high, up to $20 \%$ at 12 months, and there was no correlation found between the development of anxiety and prevalence of depression [43] in minor stroke patients. Headache after stroke is fairly common [45], however, the prevalence rate in minor stroke patients is unknown. Future studies to determine the prevalence of headaches in patients with mild stroke are necessary.

\section{Long Term Management for Minor Stroke; What Can Be Offered?}

Stroke education and patient compliance with treatment may be improved with inpatient hospitalization. In addition, close follow-up monitoring, and imaging studies in outpatients may contribute to the stratifications of patients with risk of recurrence of new stroke after TIA or minor stroke. The EXPRESS study showed [46] that up to $10 \%$ of the risk of stroke recurrence in TIA and minor stroke patients was on the first week. Early initiation of existing treatments after TIA or minor stroke was associated with an $80 \%$ reduction in the risk of early recurrent stroke. Minor stroke and TIA are typically treated conservatively with antiplatelet agents and general vascular prevention strategies. The clinical trial CHANCE demonstrates the efficacy of combination therapy of Aspirin and Clopidogrel in reducing stroke recurrence in high-risk TIA and minor stroke patients [47].

Minor stroke patients also benefit from health lifestyle education and risk factors modification, because they represent a high risk for subsequent stroke. Support services to address functional impairments, as well as assessment for 
rehabilitation [48], psychiatry follow-up if there is any indication.

\section{Conclusion}

Defining minor stroke is very challenging, considering that what can be considered mild to the physician may not be to the patients, and the degree of disability will depend on the physicians' point of view. Stroke with large vessel occlusion may sometimes present with a low NIHSS score initially, therefore, a low NIHSS score or an isolated stroke symptom should not be underestimated. To attribute the diagnoses of minor stroke there are a few things that should be considered: a) the choice of acute management; b) the symptoms characteristic; c) the short-term and medium-term outcomes; d) the possible complication in individuals' group of patients. Very little is known about the relationship of LVO, depression and cognitive disfunction in minor stroke, further studies will be necessary related to this topic. More studies should be directed to group of patients with a very mild stroke symptom to universalize the definition of a minor stroke to enhance the decision of the management in clinical protocols.

\section{Conflicts of Interest}

The authors declare no conflicts of interest regarding the publication of this paper.

\section{References}

[1] Crespi, V., Braga, M., Beretta, S., Carolei, A., Bignamini, A. and Sacco, S. (2013) A Practical Definition of Minor Stroke. Neurological Sciences, 34, 1083-1086. https://doi.org/10.1007/s10072-012-1205-8

[2] Barber, P.A., Zhang, J., Demchuk, A.M., Hill, M.D. and Buchan, A.M. (2001) Why Are Stroke Patients Excluded from TPA Therapy?: An Analysis of Patient Eligibility. Neurology, 56, 1015-1020. https://doi.org/10.1212/WNL.56.8.1015

[3] Fischer, U., Baumgartner, A., Arnold, M., Nedeltchev, K., Gralla, J., et al. (2010) What Is a Minor Stroke? Stroke, 41, 661-666. https://doi.org/10.1161/STROKEAHA.109.572883

[4] The National Institute of Neurological Disorders Stroke rt-PA Stroke Study Group (2005) Recombinant Tissue Plasminogen Activator for Minor Strokes: The National Institute of Neurological Disorders and Stroke rt-PA Stroke Study Experience. Annals of Emergency Medicine, 46, 243-252. https://doi.org/10.1016/j.annemergmed.2005.02.013

[5] Wang, D.Z., Rose, J.A., Honings, D.S., Garwacki, D.J. and Milbrandt, J.C. (2000) Treating Acute Stroke Patients with Intravenous tPA. Stroke, 31, 77-81. https://doi.org/10.1161/01.STR.31.1.77

[6] Kleindorfer, D., Kissela, B., Schneider, A., Woo, D., Khoury, J., Miller, R., Alwell, K., et al. (2004) Eligibility for Recombinant Tissue Plasminogen Activator in Acute Ischemic Stroke: A Population-Based Study. Stroke, 35, e27-e29. https://doi.org/10.1161/01.STR.0000109767.11426.17

[7] Smith, E.E., Fonarow, G.C., Reeves, M.J., Cox, M., et al. (2011) Outcomes in Mild or Rapidly Improving Stroke Not Treated with Intravenous Recombinant Tissue-Type Plasminogen Activator. Stroke, 42, 3110-3115. 
https://doi.org/10.1161/STROKEAHA.111.613208

[8] Strambo, D., Zambon, A.A., Roveri, L., Giacalone, G., Di Maggio, G., et al. (2015) Defining Minor Symptoms in Acute Ischemic Stroke. Cerebrovascular Diseases, 39, 209-215. https://doi.org/10.1159/000375151

[9] Kernan, W.N., Horwitz, R.I., Brass, L.M., Viscoli, C.M. and Taylor, K.J.W. (1991) A Prognostic System for Transient Ischemia or Minor Stroke. Annals of Internal Medicine, 114, 552-557. https://doi.org/10.7326/0003-4819-114-7-552

[10] Carlsson, G., Möller, A. and Blomstrand, C. (2004) A Qualitative Study of the Consequences of 'Hidden Dysfunctions' One Year after a Mild Stroke in Persons 75 Years. Disability and Rehabilitation, 26, 1373-1380. https://doi.org/10.1080/09638280400000211

[11] Johansson, A., Mishina, E., Ivanov, A. and Björklund, A. (2007) Activities of Daily Living among St Petersburg Women after Mild Stroke. Occupational Therapy International, 14, 170-182. https://doi.org/10.1002/oti.232

[12] Muren, M.A., Hütler, M. and Hooper, J. (2008) Functional Capacity and Health-Related Quality of Life in Individuals Post Stroke. Topics in Stroke Rehabilitation, 15, 51-58. https://doi.org/10.1310/tsr1501-51

[13] Jones, M.R., Horner, R.D., Edwards, L.J., et al. (2000) Racial Variation in Initial Stroke Severity. Stroke, 31, 563-567. https://doi.org/10.1161/01.STR.31.3.563

[14] Liu-Ambrose, T., Pang, M. Y. C. and Eng, J. J. (2007) Executive Function Is Independently Associated with Performances of Balance and Mobility in Community-Dwelling Older Adults after Mild Stroke: Implications for Falls Prevention. Cerebrovascular Diseases, 23, 203-210. https://doi.org/10.1159/000097642

[15] Dubuc, V., Choi, P., Hill, M.D. and Coutts, S.B. (2015) Can 90-Day NIHSS Be Used for Outcome Assessment in TIA and Minor Stroke Studies? Cerebrovascular Diseases, 40, 97-98. https://doi.org/10.1159/000431187

[16] Yaghi, S., Willey, J.Z., Andrews, H., Boehme, A.K., Marshall, R.S. and Boden-Albala, B. (2016) The Itemized NIHSS Scores Are Associated with Discharge Disposition in Patients With Minor Stroke. The Neurohospitalist, 6, 102-106. https://doi.org/10.1177/1941874416641466

[17] Park, T.H., Hong, K.S., Choi, J.C., et al. (2013) Validation of Minor Stroke Definitions for Thrombolysis Decision Making. Journal of Stroke and Cerebrovascular Diseases, 22, 482-490. https://doi.org/10.1016/j.jstrokecerebrovasdis.2013.03.006

[18] Yaghi, S., Herber, C., Boehme, A.K., Andrews, H., Willey, J.Z., et al. (2017) The Association between Diffusion MRI-Defined Infarct Volume and NIHSS Score in Patients with Minor Acute Stroke. Journal of Neuroimaging, 27, 388-391. https://doi.org/10.1111/jon.12423

[19] Woo, D., Broderick, J.P., Kothari, R.U., et al. (1999) Does the National Institutes of Health Stroke Scale Favor Left Hemisphere Strokes? Stroke, 30, 2355-2359. https://doi.org/10.1161/01.STR.30.11.2355

[20] Leira, E.C., Adams, H.P., Rosenthal, G.E. and Torner, J.C. (2008) Baseline NIH Stroke Scale Responses Estimate the Probability of Each Particular Stroke Subtype. Cerebrovascular Diseases, 26, 573-577. https://doi.org/10.1159/000165109

[21] Martin-Schild, S., Albright, K.C., Tanksley, J., et al. (2011) Zero on the NIHSS Does Not Equal the Absence of Stroke. Annals of Emergency Medicine, 57, 42-45. https://doi.org/10.1016/j.annemergmed.2010.06.564

[22] Adams, H.P., Davis, P.H., Leira, E.C., Bendixen, B.H., et al. (1999) Baseline NIH Stroke Scale Score Strongly Predicts Outcome after Stroke: A Report of the Trial of 
Org 10172 in Acute Stroke Treatment (TOAST). Neurology, 53, 126. https://doi.org/10.1212/WNL.53.1.126

[23] Fink, J.N., Selim, M.H., Kumar, S., Silver, B., Linfante, I., Caplan, L.R., and Schlaug, G. (2002) Is the Association of National Institutes of Health Stroke Scale Scores and Acute Magnetic Resonance Imaging Stroke Volume Equal for Patients with Rightand Left-Hemisphere Ischemic Stroke? Stroke, 33, 954-958.

https://doi.org/10.1161/01.STR.0000013069.24300.1D

[24] Wendt, M., Tütüncü, S., Fiebach, J.B., Scheitz, J.F., Audebert, H.J. and Nolte, C.H. (2013) Preclusion of Ischemic Stroke Patients from Intravenous Tissue Plasminogen Activator Treatment for Mild Symptoms Should Not Be Based on Low National Institutes of Health Stroke Scale Scores. Journal of Stroke and Cerebrovascular Diseases, 22, 550-553. https://doi.org/10.1016/j.jstrokecerebrovasdis.2013.01.021

[25] Sato, S., Uehara, T., Ohara, T., Suzuki, R., Toyoda, K. and Minematsu, K. (2014) Factors Associated with Unfavorable Outcome in Minor Ischemic Stroke. Neurolo$g y$, 83, 174-181. https://doi.org/10.1212/WNL.0000000000000572

[26] Coutts, S.B., O'Reilly, C., Hill, M.D., Steffenhagen, N., Poppe, A.Y., Boyko, M.J., et al. (2009) Computed Tomography and Computed Tomography Angiography Findings Predict Functional Impairment in Patients with Minor Stroke and Transient Ischaemic Attack. International Journal of Stroke, 4, 448-453.

https://doi.org/10.1111/j.1747-4949.2009.00346.x

[27] Rajajee, V., Kidwell, C., Starkman, S., Ovbiagele, B., Alger, J.R., Villablanca, P., Vinuela, F., et al. (2006) Early MRI and Outcomes of Untreated Patients with Mild or Improving Ischemic Stroke. Neurology, 67, 980-984. https://doi.org/10.1212/01.wnl.0000237520.88777.71

[28] Nedeltchev K., Schwegler, B., Haefeli, T., Brekenfeld, C., Gralla, J., Fischer, U., et al. (2007) Outcome of Stroke with Mild or Rapidly Improving Symptoms. Stroke, 38, 2531-2535. https://doi.org/10.1161/STROKEAHA.107.482554

[29] Nagel S., Bouslama, M., Krause, L.U., Küpper, C., Messer, M., Petersen, M., Lowens, S., Herzberg, M., et al. (2018) Mechanical Thrombectomy in Patients with Milder Strokes and Large Vessel Occlusions. Stroke, 49, 2391-2397. https://doi.org/10.1161/STROKEAHA.118.021106

[30] Powers, W.J., Rabinstein, A.A., Ackerson, T., et al. (2018) 2018 Guidelines for the Early Management of Patients with Acute Ischemic Stroke: A Guideline for Healthcare Professionals from the American Heart Association/American Stroke Association. Stroke, 49, e46-e99. https://doi.org/10.1161/STR.0000000000000158

[31] Griessenauer, C.J., Medin, C., Maingard, J., et al. (2018) Endovascular Mechanical Thrombectomy in Large-Vessel Occlusion Ischemic Stroke Presenting with Low National Institutes of Health Stroke Scale: Systematic Review and Meta-Analysis. World Neurosurgery, 110, 263-269. https://doi.org/10.1016/j.wneu.2017.11.076

[32] Rocha, M. and Jovin, T.G. (2017) Fast versus Slow Progressors of Infarct Growth in Large Vessel Occlusion Stroke. Stroke, 48, 2621-2627. https://www.ahajournals.org/doi/full/10.1161/STROKEAHA.117.017673

[33] Manno, C., Disanto, G., Bianco, G., et al. (2019) Outcome of Endovascular Therapy in Stroke with Large Vessel Occlusion and Mild Symptoms. Neurology, 93, e1618-e1626. https://doi.org/10.1212/WNL.0000000000008362

[34] Urra, X., San Román, L., Gil, F., et al. (2014) Medical and Endovascular Treatment of Patients with Large Vessel Occlusion Presenting with Mild Symptoms: An Observational Multicenter Study. Cerebrovascular Diseases, 38, 418-424. https://doi.org/10.1159/000369121 
[35] Sarraj, A., Hassan, A., Savitz, S.I., Grotta, J.C., et al. (2018) Endovascular Thrombectomy for Mild Strokes: How Low Should We Go? Stroke, 49, 2398-2405. https://doi.org/10.1161/STROKEAHA.118.022114

[36] Towfighi, A., Ovbiagele, B., Husseini, N.E., et al. (2017) Poststroke Depression: A Scientific Statement for Healthcare Professionals from the American Heart Association/American Stroke Association. Stroke, 48, e30-e43. https://doi.org/10.1161/STR.0000000000000113

[37] Hackett, M.L. and Anderson, C.S. (2005) Predictors of Depression after Stroke: A Systematic Review of Observational Studies. Stroke, 36, 2296-2301. https://doi.org/10.1161/01.STR.0000183622.75135.a4

[38] Ayerbe, L., Ayis, S., Wolfe, C.D.A. and Rudd, A.G. (2013) Natural History, Predictors and Outcomes of Depression after Stroke: Systematic Review and Meta-Analysis. The British Journal of Psychiatry: The British Journal of Psychiatry, 202, 14-21. https://doi.org/10.1192/bjp.bp.111.107664

[39] Altieri, M., Maestrini, I., Mercurio, A., Troisi, P., Sgarlata, E., Rea, V., Di Piero, V. and Lenzi, G.L. (2012) Depression after Minor Stroke: Prevalence and Predictors. European Journal of Neurology, 19, 517-521. https://doi.org/10.1111/j.1468-1331.2011.03583.x

[40] Shi, Y.Z., Xiang, Y.T., Yang, Y., Zhang, N., et al. (2015) Depression after Minor Stroke: Prevalence and predictors. Journal of Psychosomatic Research, 79, 143-147. https://doi.org/10.1016/j.jpsychores.2015.03.012

[41] Carnes-Vendrell, A., Deus, J., Molina-Seguin, J., Pifarré, J. and Purroy, F. (2019) Depression and Apathy after Transient Ischemic Attack or Minor Stroke: Prevalence, Evolution and Predictors. Scientific Reports, 9, Article No. 16248. https://doi.org/10.1038/s41598-019-52721-5

[42] Morsund, Å.H., Ellekjær, H., Gramstad, A., et al. (2019) The Development of Cognitive and Emotional Impairment after a Minor Stroke: A Longitudinal Study. Acta Neurologica Scandinavica, 140, 281-289. https://doi.org/10.1111/ane.13143

[43] Radman, N., Staub, F., Aboulafia-Brakha, T., Berney, A., Bogousslavsky, J. and Annoni, J.-M. (2012) Poststroke Fatigue Following Minor Infarcts: A Prospective Study. Neurology, 79, 1422-1427. https://doi.org/10.1212/WNL.0b013e31826d5f3a

[44] Charlotte, W., Catherine, S., Ziyah, M. and Rothwell, P.M. (2009) A Population-Based Study of the Prevalence of Fatigue after Transient Ischemic Attack and Minor Stroke. Stroke, 40, 757-761. https://doi.org/10.1161/STROKEAHA.108.527101

[45] Harriott, A.M., Karakaya, F. and Ayata, C. (2020) Headache after Ischemic Stroke: A Systematic Review and Meta-Analysis. Neurology, 94, e75-e86. https://doi.org/10.1212/WNL.0000000000008591

[46] Rothwell, P.M., et al. (2007) Effect of Urgent Treatment of Transient Ischaemic Attack and Minor Stroke on Early Recurrent Stroke (EXPRESS Study): A Prospective Population-Based Sequential Comparison. The Lancet, 370, 1432-1442. https://doi.org/10.1016/S0140-6736(07)61448-2

[47] Wang, Y.L., Pan, Y.S., Zhao, X.Q., et al. (2015) Clopidogrel with Aspirin in Acute Minor Stroke or Transient Ischemic Attack (CHANCE) Trial. Circulation, 132, 40-46. https://doi.org/10.1161/CIRCULATIONAHA.114.014791

[48] Finch, E., Foster, M., Fleming, J., et al. (2020) Exploring Changing Needs Following Minor Stroke. Health \& Social Care in the Community, 28, 347-356.

https://doi.org/10.1111/hsc. 12866 